\section{Case Reports in Ophthalmology}

Case Rep Ophthalmol 2020;11:457-465

DOI: $10.1159 / 000509263$

Published online: August 7, 2020
(C) 2020 The Author(s)

Published by S. Karger AG, Basel www.karger.com/cop

This article is licensed under the Creative Commons Attribution-NonCommercial 4.0 International License (CC BY-NC) (http://www.karger.com/Services/OpenAccessLicense). Usage and distribution for commercial purposes requires written permission.

\title{
Delayed-Onset Retrobulbar Hemorrhage and Glaucoma Drainage Device Extrusion in a Patient on Anticoagulation: A Case Report
}

\author{
Hee Jun Kimª ${ }^{a}$ Seongyong Jeong ${ }^{b}$ Su-Ho Limb, \\ ${ }^{a}$ Good Doctors Eye Hospital, Ulsan, Republic of Korea; bepartment of Ophthalmology, \\ Yeungnam University College of Medicine, Daegu, Republic of Korea; 'Department of \\ Ophthalmology, Daegu Veterans Health Service Medical Center, Daegu, Republic of Korea
}

\section{Keywords}

Retrobulbar hemorrhage - Body exposure - Ahmed glaucoma valve - Anticoagulation . Glaucoma drainage device

\begin{abstract}
Both retrobulbar hemorrhage and plate extrusion are uncommon complications after implantation of a glaucoma drainage device (GDD). There are no published reports on extrusion of an Ahmed glaucoma valve after delayed-onset retrobulbar hemorrhage in Korea. Herein, we report a case of spontaneous extrusion of a GDD after delayed-onset retrobulbar hemorrhage in a patient on anticoagulation. The case was a 72-year-old man with open-angle glaucoma and proliferative diabetic retinopathy, as well as systemic hypertension, atrial fibrillation, congestive heart failure due to coronary heart disease, and chronic kidney disease, who underwent combined Ahmed glaucoma valve implantation and cataract extraction surgery in his right eye. The body remained in position with control of intraocular pressure for 2 months. On day 67 (5 days post-coronary artery bypass surgery), he complained of decreased visual acuity, periorbital swelling, bloody discharge, and limited extraocular movement. One month later, the body was extruded with a scleral flap tear. Therefore, delayed-onset retrobulbar hemorrhage should be kept in mind in patients on anticoagulants after insertion of a GDD.
\end{abstract}




\section{Case Reports in Ophthalmology}

\section{Introduction}

Glaucoma is chronic progressive optic neuropathy with characteristic changes in the optic nerve head and visual field, and one of the leading causes of blindness worldwide [1, 2]. Although many risk factors have been identified for progression of glaucoma, including age, vascular factors, systemic disease, and genetic predisposition, elevated intraocular pressure (IOP) is widely established to be the "only" modifiable risk factor for both "onset" and "progression" of glaucoma [3].

Glaucoma drainage device (GDD) implantation has been widely used to lower IOP in eyes with complicated glaucoma, such as uveitic glaucoma, neovascular glaucoma, and pediatric or developmental glaucoma [4]. Although effective, GDD implantation has a risk of serious complications, including hypotony, flat chamber, inflammation, hyphema, valve malfunction, and tube-related problems in the Tube Versus Trabeculectomy study [5].

However, both retrobulbar hemorrhage and plate extrusion are not commonly occurring complications associated with GDD [4, 6, 7]. Moreover, a complicated case of delayed-onset retrobulbar hemorrhage and subsequent Ahmed valve extrusion has not been reported previously in Korea. Herein, we report a case of spontaneous GDD extrusion after delayed-onset retrobulbar hemorrhage in a patient on anticoagulation.

\section{Case Report}

\section{Initial Presentation}

A 72-year-old man with open-angle glaucoma and proliferative diabetic retinopathy, as well as systemic hypertension, atrial fibrillation, congestive heart failure due to coronary heart disease and chronic kidney disease, visited our clinic complaining of decreased visual acuity for several years. Two years prior to presentation, the patient had undergone uneventful phacotrabeculectomy in the left eye at another tertiary hospital to manage elevated IOP. One year prior to presentation, he underwent intravitreal bevacizumab injection in both eyes for macular edema related to diabetic retinopathy. At the initial visit, his Snellen visual acuity was 20/400 OD and 20/50 OS and the IOP was $29 \mathrm{~mm} \mathrm{Hg}$ OD and $14 \mathrm{~mm} \mathrm{Hg}$ OS with Goldmann applanation tonometry. The patient had been using topical eyedrops (brimonidine $0.15 \%$ twice a day, a fixed combination of $0.5 \%$ timolol and $2 \%$ dorzolamide twice a day, and $0.0015 \%$ tafluprost once a day) in the right eye.

Slit-lamp examination showed mild conjunctival hyperemia, a clear cornea, no sectorial iris atrophy, and no iris neovascularization or nuclear cataract in his right eye. An avascular diffuse elevated bleb was observed at the superior conjunctiva in the left eye. The pupil was round with a relative afferent pupillary defect. Gonioscopy showed wide open-angle status (Grade 4, Shaffer's classification) and mild pigmentation of the trabecular meshwork without angle neovascularization except for focal peripheral anterior synechiae in both eyes. A more densely pigmented trabecular meshwork was observed in the right eye than in the left eye (Grade 1+ OD, 2+ OS).

Ultra-widefield fundus photography revealed a clear vitreous, a slightly pale disc, a cupto-disc ratio of 0.95 with the laminar dot sign, diffuse loss of the retinal nerve fiber layer, and a panretinal photocoagulation scar due to proliferative diabetic retinopathy in both eyes. A visual field test and spectral-domain optical coherence tomography revealed advanced glaucomatous damage, with tunnel vision (mean deviation $-27.05 \mathrm{~dB}$ OD and $-29.23 \mathrm{~dB}$ OS and a respective visual field index value of 14 and 3\%) and a very thin retinal nerve fiber layer 


\section{Case Reports in Ophthalmology}

Case Rep Ophthalmol 2020;11:457-465 DOI: $10.1159 / 000509263$

C 2020 The Author(s). Published by S. Karger AG, Basel www.karger.com/cop

Kim et al.: Delayed-Onset Retrobulbar Hemorrhage and GDD Extrusion

(mean thickness: $56.52 \mu \mathrm{m}$ OD and $60.62 \mu \mathrm{m}$ OS) in both eyes (Fig. 1). Therefore, we decided on combined GDD implantation and cataract extraction surgery. The preoperative measured axial length of the right eye was $24.68 \mathrm{~mm}$ by the IOL master 500 (Carl Zeiss Meditec AG, Jena, Germany).

\section{Surgical Techniques and Early Postoperative Clinical Courses}

After application of topical anesthesia, a $2.75-\mathrm{mm}$ temporal clear corneal incision was made, and a side-port incision was made at 12 o'clock. Phacoemulsification was performed in the usual manner. Next, a foldable hydrophobic acrylic intraocular lens was implanted in the capsular bag. To prevent wound leak, the incision was hydrated and a 10-0 nylon suture was added.

A fornix-based incision is made through the superotemporal conjunctiva and Tenon's capsule. Additionally, sub-Tenon anesthesia with $2 \%$ lidocaine was administered. Tenon's capsule was dissected from the episclera as far as possible, and bleeding vessels were gently cauterized. An AGV (FP7 model; New World Medical, Rancho Cucamonga, CA, USA) was inserted into the pocket between the rectus muscles. We did not use mitomycin-C or anti-metabolites. The body of the AGV was positioned $8 \mathrm{~mm}$ from the limbus, and then fixed to the sclera with an 8-0 nylon suture. A drainage tube with a bevel-up angle of $30^{\circ}$ was entered at 1-2 $\mathrm{mm}$ from the limbus and fixed at the sclera using a "figure of eight" suture. The exposed drainage tube was covered with a partial thickness scleral flap using an envelope technique. In the final step, the conjunctiva was anchored to the limbus with an absorbable 8-0 Vicryl suture [4] (Fig. 2).

The body of the AGV remained in position with control of IOP (8-13 mm Hg without medication) and no hypertensive episodes for 2 months. Visual acuity was improved from 20/400 to $20 / 40$ by 1 month postoperatively (Fig. 3 ). There was no visible conjunctival abnormality during the early postoperative phase.

\section{Delayed-Onset Retrobulbar Hemorrhage and Body Exposure}

At 2 months postoperatively, the patient experienced chest pain and was diagnosed to have triple-vessel ischemic heart disease. Therefore, he underwent coronary artery bypass surgery and intensive anticoagulation treatment. His medication included aspirin, clopidogrel, and rivaroxaban.

On day 67 (5 days post-coronary artery bypass surgery), he complained of periorbital swelling, bloody discharge, and limited extraocular movement. He denied the history of rubbing or trauma except coughing. Physical examination revealed an erythematous swollen right eyelid and significant proptosis with limitation of horizontal gaze. His visual acuity was decreased to $20 / 200$ and his IOP was $36 \mathrm{~mm} \mathrm{Hg}$.

Slit-lamp examination showed bloody discharge, massive subconjunctival hemorrhage in the superotemporal area, and a relatively intact conjunctival epithelium. The anterior chamber was deep without hyphema (Fig. 4a, b). Fundus examination showed a flat retina and a relatively clear vitreous without subhyaloid or suprachoroidal hemorrhage. Ocular pain, increased pressure, decreased visual acuity, relatively afferent pupillary defect, subconjunctival hemorrhage, eyelid ecchymosis, and limitation of extraocular muscle suggested the retrobulbar hemorrhage clinically. However, the patient declined to undergo computerized tomography (CT) scan. He received two tablets of acetazolamide $(250 \mathrm{mg})$ and topical glaucoma medication, including brimonidine and fixed-combination timolol and dorzolamide eyedrops. His IOP stabilized to the high-teen level (17-19 mm Hg) during 1 month after bleeding. 


\section{Case Reports in Ophthalmology}

One month later, slit-lamp examination revealed an anteriorly migrated plate (body) and tube, peaking of the pupil, and a scleral flap tear. Therefore, we planned surgery for repositioning of the GDD (Fig. 4c, d). Unfortunately, 3 days later, he developed markedly decreased visual acuity and ocular pain, and the plate body was found to be expelled (Fig. 4e, f). We explanted the GDD, performed a wound culture, administered intravitreal and intracameral antibiotic injections, and irrigated the anterior chamber. Microbial examination showed growth of gram-negative rods (Pseudomonas aeruginosa). Fortified antibiotic (5\% ceftazidime and $2 \%$ amikacin) eyedrops were used to control secondary infection associated with body exposure. Two weeks after antibiotic injections, although the anterior chamber and vitreous cavity were clear, his IOP increased again to $30 \mathrm{~mm} \mathrm{Hg}$ (Fig. 5). Therefore, we planned trabeculectomy in the superonasal quadrant as further treatment.

\section{Discussion}

Various complications have been reported following glaucoma implant surgery [4,5]. The common early postoperative complications include hypotony, a flat chamber, choroidal effusion, and suprachoroidal hemorrhage. In contrast, late postoperative complications are more likely to be tube block, migration, implant exposure, infection, and corneal decompensation $[4,5]$. Most cases of retrobulbar hemorrhage following GDD implant surgery are related to retrobulbar anesthesia in the early postoperative phase. Delayed-onset retrobulbar hemorrhage is rare, and one study hypothesized that improper positioning of the implant could damage the posterior ciliary arteries and nearby vessels [6, 7].

In our case, 5 days after coronary artery bypass surgery, the patient complained of periorbital swelling, bloody discharge, massive subconjunctival hemorrhage, and limited extraocular movement. One month later, the body was extruded with a scleral flap tear.

Hemodynamic instability with intensive use of anticoagulants might induce increasing bleeding tendency and vulnerable vessels. Despite anchoring the implant to the sclera tightly, slightly horizontal movement of the GDD body on that vascular predisposition, could irritate the vulnerable vessels, cause the bleeding, and aggravate the plate instability by volume effect. Finally, hematoma or massive hemorrhage pushing the AGV might induce or exacerbate the tearing of scleral flap and subsequent extrusion of GDD. Considering the history of coughing after coronary artery bypass surgery, Valsalva maneuver might also contribute to a possible etiologic factor. Alternative explanations for the massive bleeding include trauma or initially improper plate fixation secondary to compromised scleral resistance, low scleral rigidity, or insufficient viscoelastic material utilized in the anterior chamber [8]. Although these explanations are plausible, a fibrous capsule usually forms near the GDD about 4-6 weeks after surgery and regulates flow. Therefore, these possibilities are less likely.

Optimal positioning of GDD in relation to the limbus and optic nerve is crucial in glaucoma implant surgery [9]. The body of the AGV was fixed $8 \mathrm{~mm}$ from the limbus in this patient. The length of the body (front to back) of the Ahmed FP7 model is $16 \mathrm{~mm}$. Kahook et al. [9] reported that the measured distances from the limbus to the anterior plate border varied according to axial lengths. In their study, measured distances from the limbus to the anterior plate border were $9.0 \mathrm{~mm}$ for $22.5 \mathrm{~mm}, 10.5 \mathrm{~mm}$ for $24.5 \mathrm{~mm}$, and $11.5 \mathrm{~mm}$ for $26 \mathrm{~mm}$, while remaining 2 $\mathrm{mm}$ away from the optic nerve. In this context, posterior placement of the plate is desirable to decrease the potential for anterior exposure [9]. Although the body of the AGV was positioned $8 \mathrm{~mm}$ from the limbus in our patient, relatively anterior location of the plate might affect the extrusion of the implant, considering the axial length of $24.68 \mathrm{~mm}$. The 8-mm distance from 


\section{Case Reports in Ophthalmology}

Kim et al.: Delayed-Onset Retrobulbar Hemorrhage and GDD Extrusion

the limbus is a generally acceptable location; however, an extraordinary situation of the patient with systemic risk factors may contribute to the occurrence of the hemorrhage or extrusion. On the contrary, significant optic nerve complications may occur if the GDD is implanted too posteriorly. Thus, the ophthalmologists should consider the optimal position of GDD in relation to limbus and optic nerve head based on the clinical profile of each patient.

Our case was notable for extrusion of the GDD body after delayed-onset retrobulbar hemorrhage following successful implantation of an AGV. We believe that massive bleeding around the plate and the secondary flap tear due to the volume effect of hemorrhage played a major role in tissue fragility and that loosening of the plate suture changed the position of the plate until complete extrusion occurred $[6,8]$.

Imaging studies including CT scan or MRI images to confirm the diagnosis of retrobulbar hemorrhage are usually recommended [10]. CT scans are preferred because of their fast acquisition time and better visualization of the bony anatomy. However, the patient declined to undergo a CT scan. This is a limitation of this case report.

In conclusion, we have encountered a patient with delayed-onset retrobulbar hemorrhage after intensive anticoagulation who developed the rare complication of postoperative body extrusion. During the acute phase of bleeding, the GDD was not exposed and relatively favorable IOP control was obtained with medical treatment. However, he presented later with spontaneous GDD extrusion. To the best of our knowledge, this is the first case of spontaneous GDD extrusion secondary to delayed-onset retrobulbar hemorrhage following GDD implantation in Korea. Delayed-onset retrobulbar hemorrhage should be kept in mind in patients on anticoagulants after insertion of a GDD.

\section{Acknowledgments}

We would like to thank Editage (www.editage.co.kr) for English language editing.

\section{Statement of Ethics}

This case study was conducted in accordance with the Declaration of Helsinki. Written informed consent was obtained from the patient for publication of this case report and any accompanying images. A copy of the written consent is available for review by the Editors.

\section{Disclosure Statement}

The authors have no conflicts of interest to declare.

\section{Funding Sources}

No funding was received. 


\section{Case Reports in Ophthalmology}

\section{Author Contributions}

Data curation: Hee Jun Kim, Seongyong Jeong, and Su-Ho Lim. Methodology: Su-Ho Lim. Supervision: Su-Ho Lim. Surgery: Su-Ho Lim. Validation: Hee Jun Kim, Seongyong Jeong, and Su-Ho Lim. Writing and review: Hee Jun Kim, Seongyong Jeong. and Su-Ho Lim.

\section{References}

1 Quigley HA, Broman AT. The number of people with glaucoma worldwide in 2010 and 2020. Br J Ophthalmol. 2006 Mar;90(3):262-7.

2 Tham YC, Li X, Wong TY, Quigley HA, Aung T, Cheng CY. Global prevalence of glaucoma and projections of glaucoma burden through 2040: a systematic review and meta-analysis. Ophthalmology. 2014 Nov;121(11):2081-90.

3 Leske MC, Heijl A, Hussein M, Bengtsson B, Hyman L, Komaroff E; Early Manifest Glaucoma Trial Group. Factors for glaucoma progression and the effect of treatment: the early manifest glaucoma trial. Arch Ophthalmol. 2003 Jan;121(1):48-56.

4 Riva I, Roberti G, Oddone F, Konstas AG, Quaranta L. Ahmed glaucoma valve implant: surgical technique and complications. Clin Ophthalmol. 2017 Feb;11:357-67.

5 Gedde SJ, Herndon LW, Brandt JD, Budenz DL, Feuer WJ, Schiffman JC, et al. Postoperative complications in the Tube Versus Trabeculectomy (TVT) study during five years of follow-up. Am J Ophthalmol. 2012 May;153(5):804-14e1.

6 Chan CH, Lai JS, Shen SY. Delayed retrobulbar haemorrhage after Ahmed glaucoma implant: a case report. Eye (Lond). 2006 Apr;20(4):494-5.

7 Fry R, Ring P. Delayed retrobulbar haemorrhage associated with a repeat sub-Tenon's block. Anaesth Intensive Care. 2008 Sep;36(5):752-3.

8 AlDarrab A, AlBahlal A, Dibaji M, AlZaid A, AlJadaan I, Elkhamary S, et al. Spontaneous glaucoma drainage device extrusion after early postoperative orbital cellulitis - Case report and literature review. Saudi J Ophthalmol. 2019 Apr-Jun;33(2):192-5.

9 Kahook MY, Noecker RJ, Pantcheva MB, Schuman JS. Location of glaucoma drainage devices relative to the optic nerve. Br J Ophthalmol. 2006 Aug;90(8):1010-3.

10 Winterton JV, Patel K, Mizen KD. Review of management options for a retrobulbar hemorrhage. J Oral Maxillofac Surg. 2007 Feb;65(2):296-9.

Hee Jun Kim and Seongyong Jeong contributed equally to this article as first authors. 
Case Reports in Ophthalmology
Case Rep Ophthalmol 2020;11:457-465 DOI: $10.1159 / 000509263$

(c) 2020 The Author(s). Published by S. Karger AG, Basel www.karger.com/cop

Kim et al.: Delayed-Onset Retrobulbar Hemorrhage and GDD Extrusion

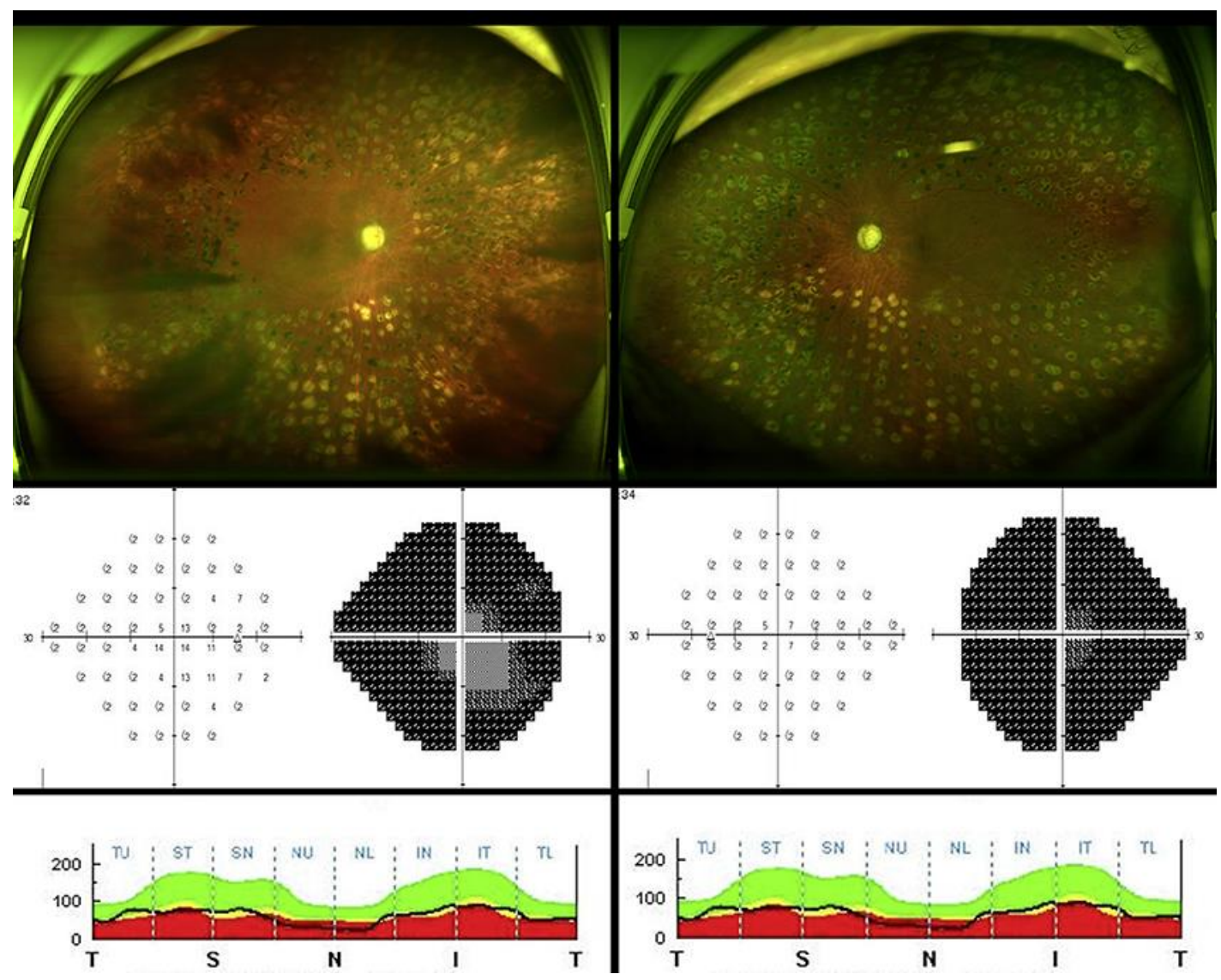

Fig. 1. Preoperative ultra-widefield fundus images, visual fields, and optical coherence tomography. 
Case Reports in Ophthalmology

\begin{tabular}{l|l}
\hline Case Rep Ophthalmol 2020;11:457-465 \\
\hline DOI: 10.1159/000509263 & $\begin{array}{l}\text { @ 2020 The Author(s). Published by S. Karger AG, Basel } \\
\text { www.karger.com/cop }\end{array}$ \\
\hline
\end{tabular}

Kim et al.: Delayed-Onset Retrobulbar Hemorrhage and GDD Extrusion

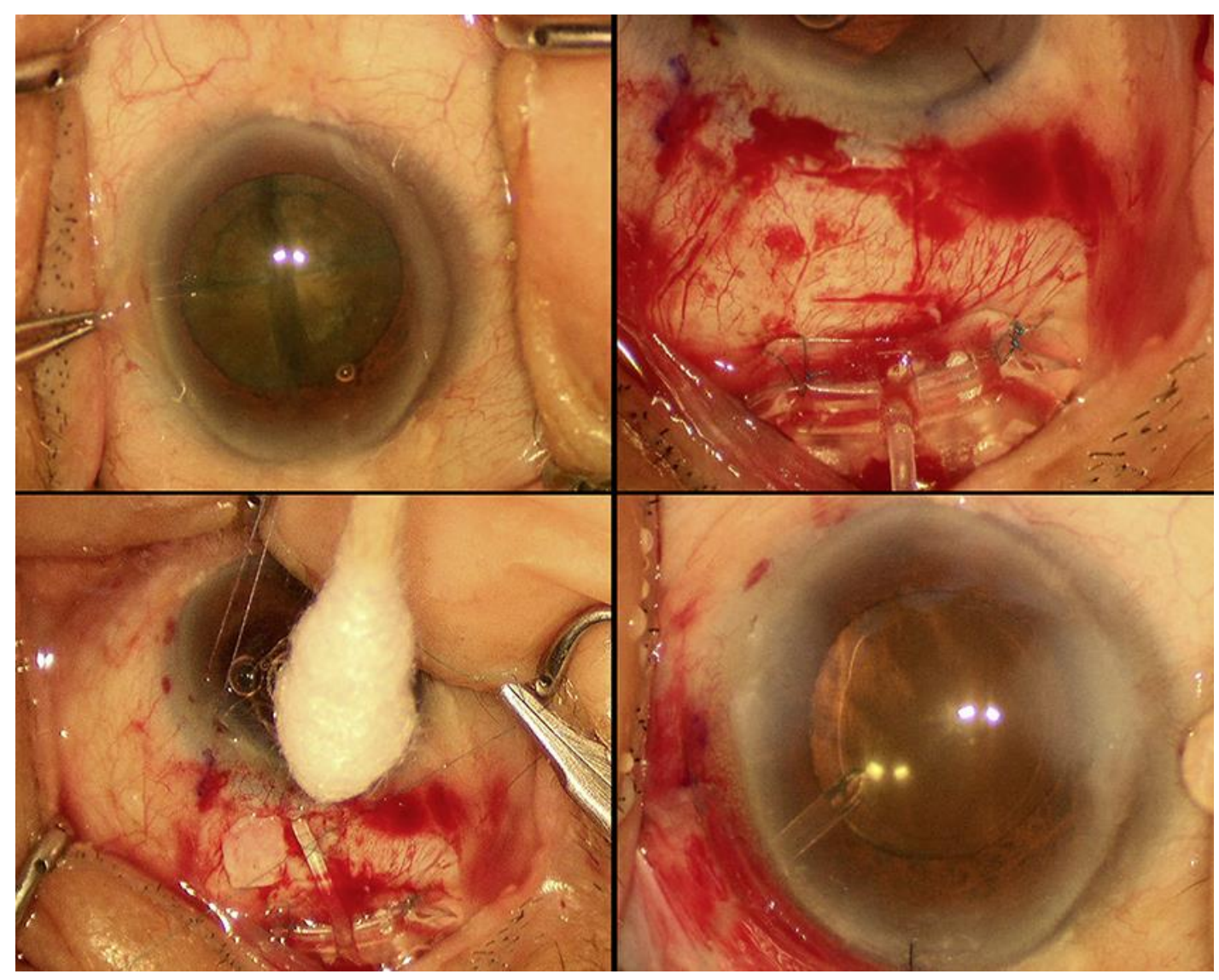

Fig. 2. Intraoperative findings for Ahmed valve implantation under a surgical microscope.

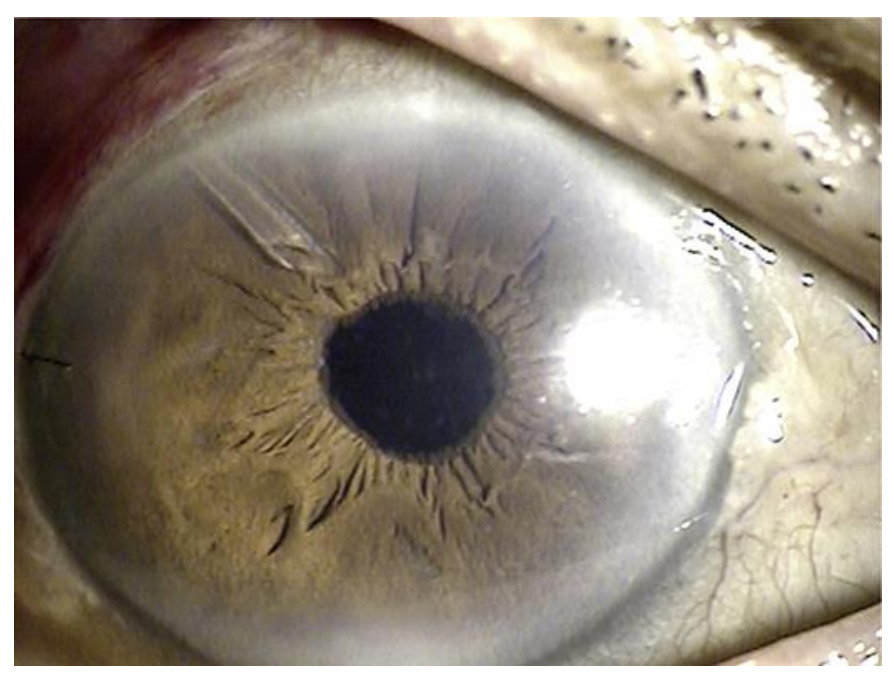

Fig. 3. Slit-lamp examination 7 days after Ahmed valve implantation. 


\section{Case Reports in Ophthalmology}
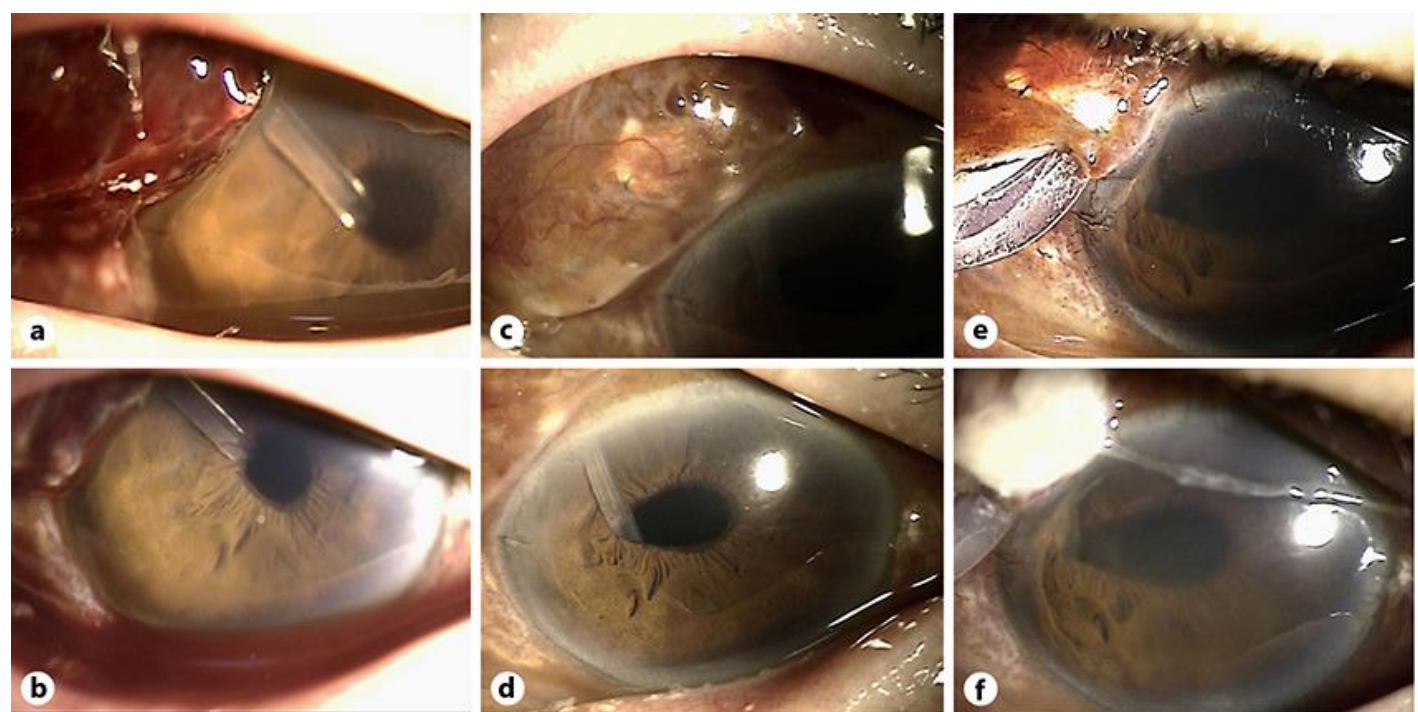

Fig. 4. Delayed-onset retrobulbar hemorrhage and body exposure. Slit-lamp examination demonstrated a massive subconjunctival hemorrhage and bloody discharge (a, b). An anteriorly migrated plate and tube, peaking of the pupil, and a scleral flap tear were observed 1 month later $(\mathbf{c}, \mathbf{d})$. Three days later, extrusion of the glaucoma drainage plate and mucopurulent discharge were observed $(e, f)$.

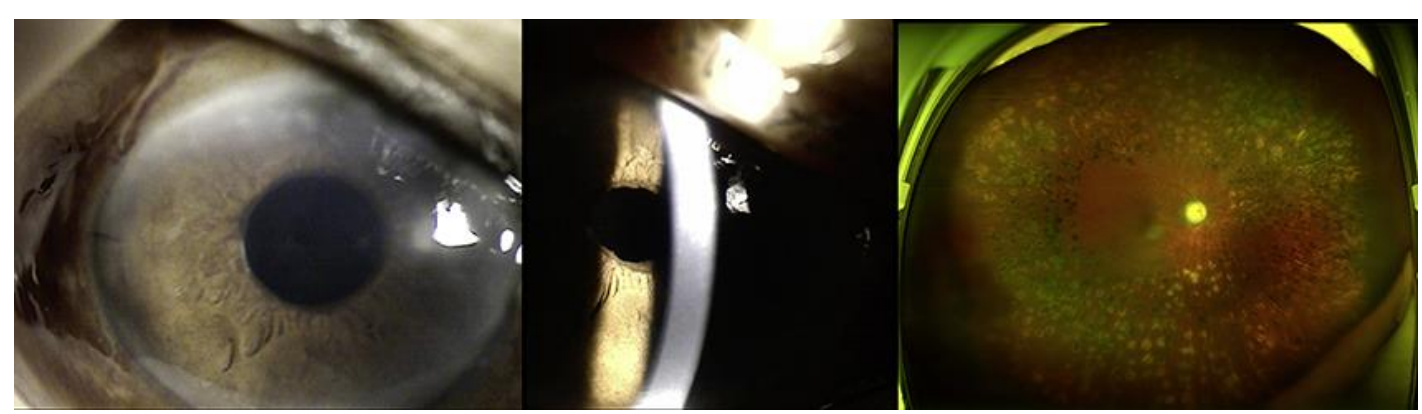

Fig. 5. Slit-lamp examination and ultra-widefield fundus photography findings 1 month after removal of the exposed Ahmed glaucoma valve. 